\title{
PERFORMANCE AND ECOLOGICAL OBJECTIVE INVESTIGATION OF TWO- STATE IRREVERSIBLE QUANTUM HEAT ENGINE
}

\author{
Emin Açıkkalp ${ }^{1, *}$, Mohammad H. Ahmadi²
}

\begin{abstract}
This paper considers irreversible two-state quantum Carnot heat engine. Basic thermodynamic parameters including power output and energy efficiency are considered, besides ecological function. Ecological function gives someone information about balance between power output and exergy destruction. The results show that ecological function have maximum (optimum) point for a and there is no optimum point for any parameter for $\mathrm{x}$ and $\mathrm{y}$. All parameters are compared with each other and the most convenient operation conditions are recommended.
\end{abstract}

\section{Keywords: Quantum Heat Engine, Irreversibility, Ecological Function}

\section{INTRODUCTION}

Since Sadi Carnot presented first reversible heat engine model, scientists and engineers have tried to develop new models and new heat engines. Classical heat engines convert heat energy taken from a heat source to work output. Carnot engine provides upper limits for heat engines and all heat engines efficiency must be smaller than Carnot heat engine. This means it has no irreversibility or thermodynamic loss.

Development in the quantum physics and quantum thermodynamics has been gained the attention on the quantum heat engines. Different from the classical heat engines, quantum heat engines use quantum matter as a working fluid and they obey the law of quantum mechanics. In the literature some examples of the quantum, molecular and nano heat engines and quantum thermodynamics can be found [1-27].

In this paper an irreversible two-state Quantum Carnot Engine is taken into account. A quantum heat engine is described and an irreversible model is designed. Besides basic thermodynamics including power output and efficiency, ecological function presented by Angulo-Brown and improved by Yan, is investigated too [28,29]. Ecological function is very useful method to optimize thermal cycles, because, it provides maximum power output and exergy destruction difference and some recent applications of the ecological function can be found in [30-32]. All parameter is obtained as dimensionless, results are presented and recommendations have been made.

\section{ANALYSIS}

For a simple quantum system of a particle mass confined to one-dimensional square-well of width L, time independent Schrödinger equation is:

$$
\frac{d^{2} \Psi}{d x^{2}}+\frac{2 m E}{\hbar^{2}} \Psi=0
$$

where, $\mathrm{m}$ is the mass of the particle and $\mathrm{E}$ is the energy of the particle. the wave function is $\Psi(0)=0$ and $\Psi(\mathrm{L})=0$ and the defined as eq. (2).

$$
\begin{aligned}
& \text { is } \Psi(0)=0 \text { and } \Psi(\mathrm{L})=0 \\
& \Psi(x)=\sum_{n=1}^{\infty} a_{n} \phi_{n}(x)
\end{aligned}
$$

Boundary conditions of $\Psi(\mathrm{x})$ wave function is where $\mathrm{a}_{\mathrm{n}}$ satisfy the orthonormal condition and $\phi_{\mathrm{n}}(\mathrm{x})$ is:

$$
\phi_{n}(x)=\sqrt{\frac{2}{L}} \sin \left(\frac{n \pi}{L} x\right)
$$

\footnotetext{
This paper was recommended for publication in revised form by Regional Editor Tolga Taner ${ }^{1}$ Department of Mechanical Engineering, Engineering Faculty, Bilecik S.E. University, Bilecik, Turkey ${ }^{2}$ Faculty of Mechanical Engineering, Shahrood University of Technology, Shahrood, Iran *E-mail address: eacikkalp@gmail.com, emin.acikkalp@bilecik.edu.tr Orcid id: 0000-0001-5356-1467, 0000-0002-5488-250X 
Normalization condition is:

$$
\sum_{n=1}^{\infty}\left|a_{n}\right|^{2}=\sum_{n=1}^{\infty} p_{n}=1
$$

These eigenfunctions are associated with the eigenenergies:

$$
E_{n}=\frac{n^{2} \pi^{2} \hbar^{2}}{2 m L^{2}}
$$

and energy of the system is:

$$
E=\sum_{n=1}^{\infty}\left|a_{n}\right|^{2} E_{n}=\sum_{n=1}^{\infty} p_{n} E_{n}
$$

Supposing infinite walls of potential well, say the wall at $\mathrm{x}=\mathrm{L}$, is allowed to move an infinitesimal amount $\mathrm{dL}$ then the wave function $\varphi(\mathrm{x})$, eigen functions $\varphi_{\mathrm{n}}(\mathrm{x})$ and energy eigenvalues $\mathrm{E}_{\mathrm{n}}$ all vary infinitesimally as function of L. In this situation, it is natural to define the force on the wall of the potential well as the negative derivative of the energy [4]:

$$
F=-\frac{d E}{d L}
$$

Work done by the system is shown in eq. (8):

$$
W=\int_{L_{1}}^{L_{2}} F d L
$$

An irreversible Carnot quantum heat engine is considered. Total internal energy of the system remains constant. In classical thermodynamics, an isoenergetic process and an isothermal process are same for the ideal gas. However, it is not always true for the quantum system. Fortunately, when the quantum degeneracy effect [3] is ignored, these two processes are same for particle in $1 \mathrm{D}$ box in this paper. F-L diagram of the quantum Carnot engine is shown in fig. $1.1 \longrightarrow 2$ process and $3 \longrightarrow 4$ process are heat exchange processes with the heat reservoirs. These processes are isothermal and heat input (W) (occurred in temperature $\mathrm{T}_{\mathrm{H}}$ ) and heat rejection (W) (occurred in temperature $\mathrm{T}_{\mathrm{L}}$ ) can be described as eq. (9) and (10) respectively [3]:

$$
\begin{gathered}
Q_{H}=\frac{\pi^{2} \hbar^{2}}{m L_{1}^{2}}\left(4-3 p_{12}\right) \ln (x) \varepsilon_{H} \\
Q_{L}=\frac{\pi^{2} \hbar^{2}}{m a L_{3}^{2}}\left(4-3 p_{32}\right) \ln (y) \varepsilon_{L}
\end{gathered}
$$

where $\varepsilon_{\mathrm{H}}$ and $\varepsilon_{\mathrm{L}}$ are the heat transfer effectiveness, $\mathrm{x}=\mathrm{L}_{2} / \mathrm{L}_{1}$ and $\mathrm{y}=\mathrm{L}_{3} / \mathrm{L}_{4}$. One, using second law of the thermodynamics, can yield eq. (11):

$$
I \frac{Q_{L}}{T_{L}}=\frac{Q_{H}}{T_{H}}
$$

where I is the irreversibility parameter and it is between 0 and $1 . T_{L}$ and $T_{H}$ are, temperature of the heat sink and heat source temperature respectively. using eq. (9) and (11) one can yield heat rejection (J) as: 


$$
Q_{L}=\frac{\pi^{2} \hbar^{2} \ln (x)\left(3 p_{12}-4\right) \varepsilon_{H} z}{I m L_{1}^{2}}
$$

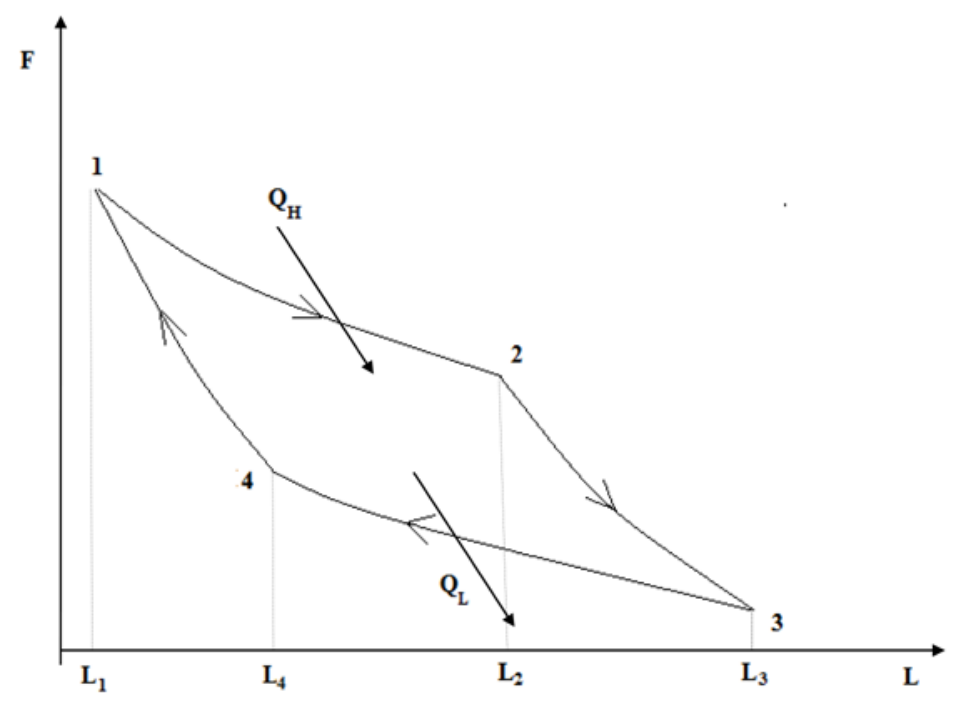

Figure 1. F-L diagram of quantum Carnot cycle

and equalizing eq. (10) and (12), irreversibility parameter is calculated as follows:

$$
I=\frac{\operatorname{aln}(x) \varepsilon_{H}\left(3 p_{12}-4\right) z}{\varepsilon_{L} \ln (y)\left(3 p_{32}-4\right)}
$$

where , $\mathrm{a}=\mathrm{L}_{3} / \mathrm{L}_{1}, \mathrm{z}=\mathrm{T}_{\mathrm{L}} / \mathrm{T}_{\mathrm{H}}$. Using the first law of the thermodynamics work output of the system is:

$$
W=Q_{H}-Q_{L}
$$

Using eqs. (9) and (12), work output is (J):

$$
W=\frac{\hbar^{2} \pi^{2}\left(a \varepsilon_{H} \ln (x)\left(4-3 p_{12}\right)+\varepsilon_{L} \ln (y)\left(3 p_{32}-4\right)\right)}{a L_{1}^{2} m}
$$

and power output is (W):

$$
P=\frac{W}{t}=-\frac{\hbar^{2} \pi v\left(a \varepsilon_{H}\left(3 p_{12}-4\right) \ln (x)+\varepsilon_{L}\left(4-3 p_{32}\right) \ln (y)\right)}{2(a-1) a L_{1}^{3} m}
$$

where, $\mathrm{t}$ is the cycle period and described as [1]:

$$
t=\frac{2 L_{1}(a-1)}{v}
$$

Energy efficiency of the system is:

$$
\eta=\frac{W}{Q_{H}}
$$

and exergy destruction rate is (W): 


$$
E x d=\frac{\left(Q_{H} k(1-\eta-z)\right)}{t}
$$

where $\mathrm{k}=\mathrm{T}_{\mathrm{o}} / \mathrm{T}_{\mathrm{L}} \mathrm{T}_{\mathrm{o}}$ is ambient temperature. Finally, ecological function $(\mathrm{W})$ is defined as:

$$
E C=P-E x d
$$

In this research all investigated parameters are made dimensionless by dividing $\frac{\pi^{2} \hbar^{2} \mathrm{v}}{\mathrm{mL}_{1}^{3}}$, $\mathrm{p}$ is the dimensionless power output, exd is the dimensionless exergy destruction and ec is the dimensionless ecological function. These parameters are listed as follows respectively:

$$
\begin{gathered}
p=\frac{a \varepsilon_{H}\left(4-3 p_{12}\right) \ln (x)+\varepsilon_{L}\left(3 p_{32}-4\right) \ln (y)}{2(a-1) a} \\
\text { exd }=\frac{k\left(\varepsilon_{L}\left(4-3 p_{32}\right) \ln (y)+\left(a z \varepsilon_{H}\left(4-3 p_{12}\right) \ln (x)\right)\right.}{2(a-1) a} \\
e c=\frac{\varepsilon_{L}\left(3 p_{32}-4\right) \ln (y)(1+t)-a \varepsilon_{H}\left(3 p_{12}-4\right) \ln (x)(1+k z)}{2(a-1) a}
\end{gathered}
$$

\section{RESULTS AND DISCUSSION}

Parameters used in calculations are $\varepsilon_{\mathrm{H}}=\varepsilon_{\mathrm{L}}=0.9, \mathrm{z}=0.17, \mathrm{k}=1.2, \mathrm{a}=3, \mathrm{y}=2, \mathrm{x}=2$ for the irreversible two-state quantum heat engine.

Power and ecological function changes are shown in figure 2. according to a. As it seen, power output decreases logarithmically with a and this decreasing range is about $82 \%$. Ecological function has maximum (optimum) point which is at $a=4.3$ and equal to 0.144 . Negative values of the ecological function is resulted from that exergy destruction value are bigger than power output value at this point. In figure 3 , it is shown variation of energy efficiency and exergy destruction with a. Exergy destruction and energy efficiency have no optimum points and they change logarithmically. Exergy destruction decreases dramatically until about 1.5 and after this

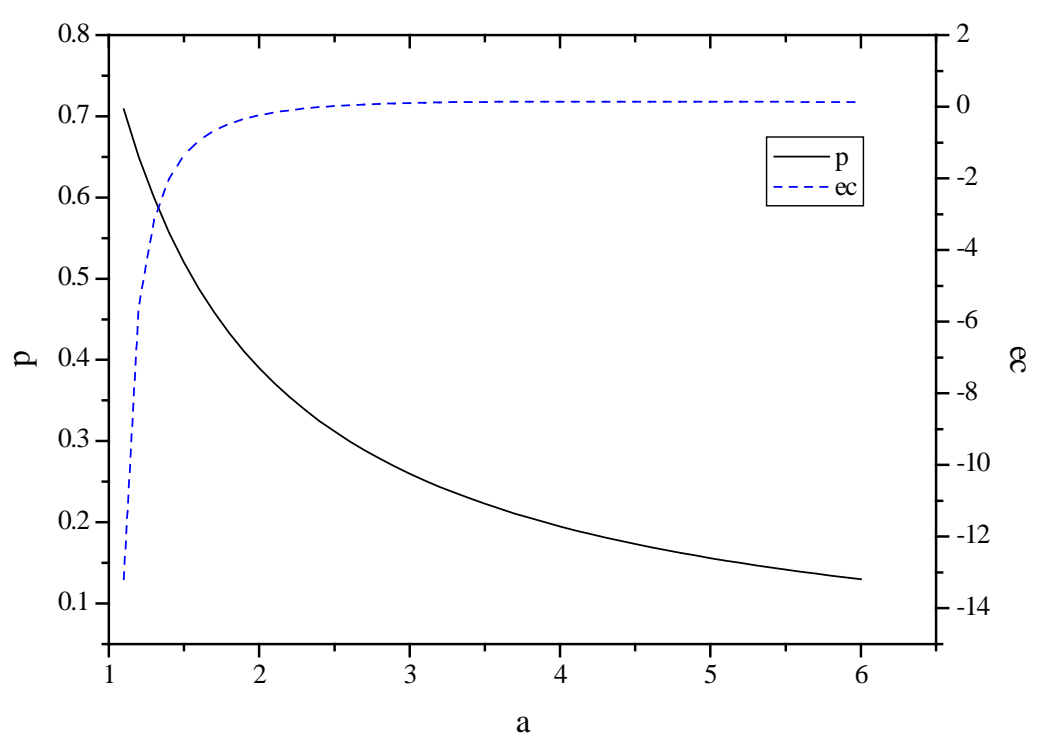

Figure 2. Variation of $\mathrm{p}$ and ec with a 
point, values change so slowly and it is nearly linear. Energy efficiency increases and this increasing rate is nearly $90 \%$. At the maximum ecological point, power output is equal to 0.181 which is $26 \%$ of the its maximum, which is obtained at the $\mathrm{a}=1.3$, at the maximum ecological function point, energy efficiency is $76 \%$ and the exergy destruction is 0.037. Therefore, a should be chosen at point where ecological function is maximum, this provides high energy efficiency and low exergy destruction but low power output also. In the ecological function, difference between power output and exergy destruction is maximum, which provides a comparison among them.

In figure 4, one can see change of power output, ecological function, exergy destruction with x. None of the investigated parameters has optimum point. In addition, all of them vary logarithmically, however, variation of power output, exergy destruction, ecological function are nearly linear. Power output increases with $\mathrm{x}$ and this increasing rate is about $97 \%$. Similarly energy efficiency rises up with $\mathrm{x}$ and it increases very fast until $\mathrm{x}=2$ after this point, variation of it is nearly linear. Increasing of energy efficiency rate is equal to 86\%. Finally, ecological function rises up with $\mathrm{x}$ and according to results, $\mathrm{x}$ value should be chosen as big as possible. Because, at the big $x$ values, power output, energy efficiency and ecological function have bigger values, while exergy destruction has smaller values.

In figure 5, change of power output, ecological function and exergy destruction with y can be shown. In contrast to previous results, exergy destruction, power output and energy efficiency reduce, while ecological function rises up. All of the considered parameters change nearly linear and none of them has no optimum point. Change at the power output, efficiency, exergy destruction are $72 \%, 72 \%$ and $95 \%$ respectively. At the small values, ecological function, power output and energy are bigger, while exergy destruction has smaller values, that's why, y should be chosen as small as possible.

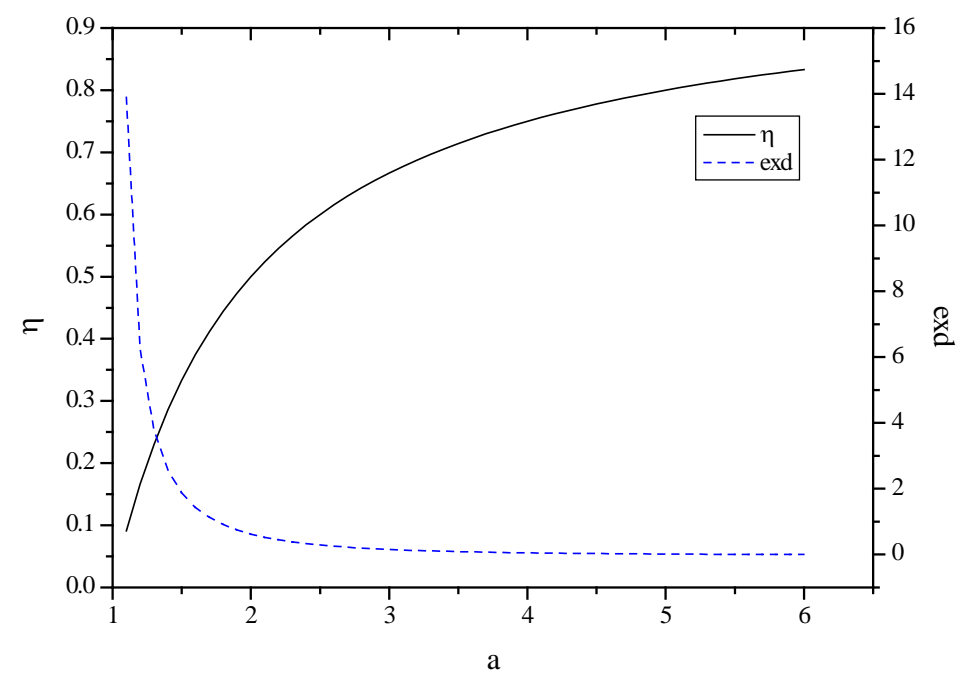

Figure 3. Variation of $\eta$ and exd with a

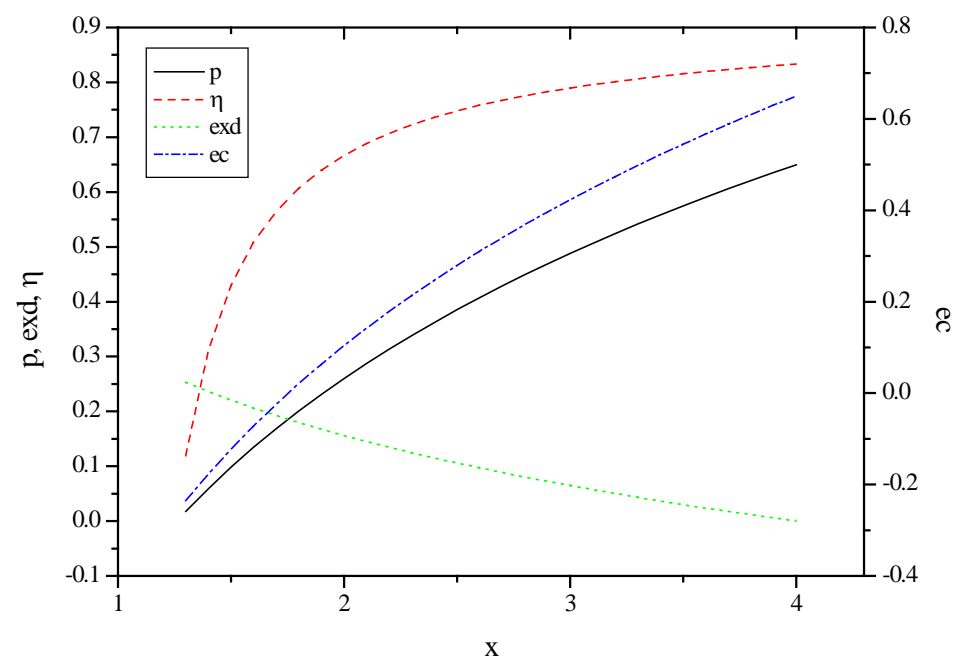

Figure 4. Variation of $p$, exd, $\eta$ and ec with $x$ 


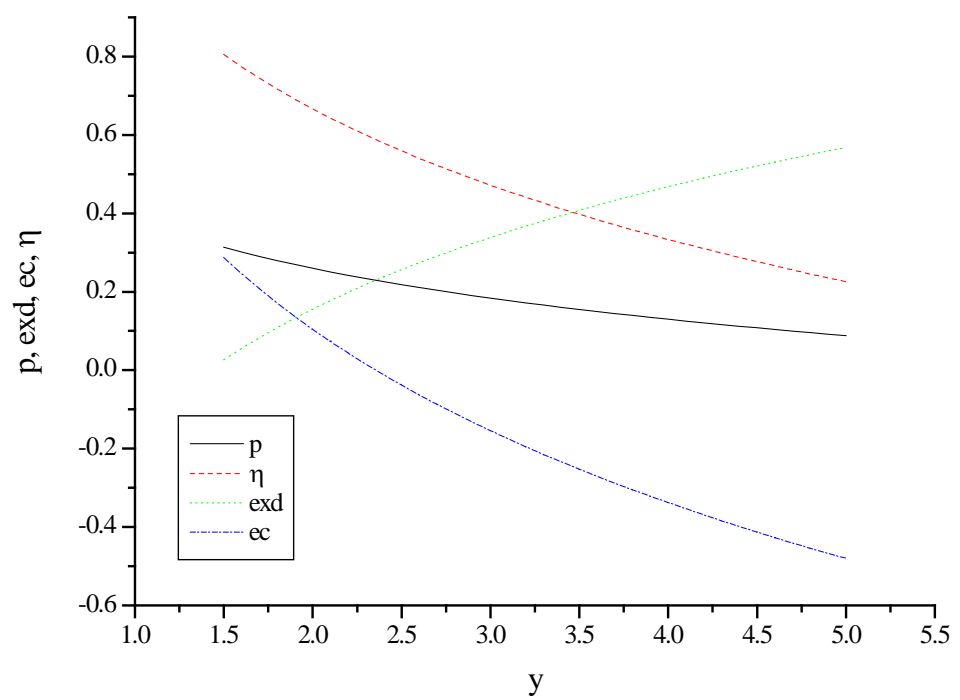

Figure 5. Variation of $p$, exd, $\eta$ and ec with $y$

\section{CONCLUDING REMARKS}

This paper is about investigating irreversible quantum Carnot cycle. Addition to the basic parameters, which include power output, energy and exergy efficiencies, ecological function is researched too. Results show that a should be chosen at the optimum ecological function value and $\mathrm{x}$ should be chosen as big as possible in contrast to $y$. The maximum power output obtained is 0.709 , the maximum energy efficiency is $83 \%$ respectively, in addition, the maximum ecological function is 0.650 in all results and all parameters considered. Finally, it can be said that quantum or micro/nano thermal system should be focused on at the future, because, in last decades, there has been some important developments in the nanotechnology field. In addition using some different evaluation criteria is recommended for quantum- micro/nano cycles.

\section{ACKNOWLEDGEMENTS}

Authors would like to thank the reviewers for their valuable comments, which have been utilized in improving the quality of the paper.

\section{REFERENCES}

[1] Abe S. Maximum-power quantum-mechanical Carnot engine. Physical Review E 2011; 83: 041117. doi: 10.1103/PhysRevE.83.041117.

[2] Bender CM., Brody D.C., Meister B.K.. Quantum mechanical Carnot engine. J. Phys. A: Math. Gen. 2000; 33: 4427-4436. doi:10.1088/0305-4470/33/24/302.

[3] Yin Y., Chen L., Wu F. Optimal power and efficiency of quantum Stirling heat engines. Eur. Phys. J. Plus 2017; 132: 45. doi: 10.1140/epjp/i2017-11325-0.

[4] Latifah E., Purwanto A. Multiple-State Quantum Carnot Engine. Journal of Modern Physics 2011;2: 13661372. doi:10.4236/jmp.2011.211169.

[5] Liu X., Chen L., Wu F., Sun F. Fundamental optimal relation of an irreversible quantum Carnot heat pump with spin-1/2 systems. Mathematical and Computer Modeling 2011;54: 190-202. doi: doi.org/10.1016/j.mcm.2011.02.001.

[6] Liu X., Chen L., Wu F., Sun F., Cooling load and energy efficiency optimization of an irreversible Carnot refrigerator with spin-1/2 systems. International Journal of Energy and Environment 2011; 2: 797-812.

[7] Feldmann, T., Kosloff, R. Performance of Discrete Heat Engines and Heat Pumps in Finite Time. Phys.Rev. E 2000; 61: 4774-4790. doi: 10.1103/PhysRevE.61.4774.

[8] Liu X., Chen L., Wu F., Sun F. Ecological optimization of an irreversible quantum Carnot heat engine with spin-1/2 systems. Physica Scripta 2010; 81:025003. doi.org/10.1088/0031-8949/81/02/025003.

[9] Geva, E., Kosloff, R. A quantum-mechanical heat engine operating in finite time. a model consisting of spin1/2 systems as working fluid. J. Chem. Phys. 1992;96: 3054-3067. doi: 10.1063/1.461951.

[10] Wu, F., Chen, L.G., Sun, F.R. and Wu, C. Performance of an irreversible quantum Carnot engine with spin1/2. J. Chem. Phys. 2006; 124: 214702. doi:10.1063/1.2200693.

[11] Beretta G.P. Quantum thermodynamic Carnot and Otto-like cycles for a two-level system. EPL 2012; 99: 20005. doi: 10.1209/0295-5075/99/20005.

[12] Henrich M.J., Rempp F., Mahler G., Quantum thermodynamic Otto machines: A spin-system approach. Eur. Phys. J. Special Topics 2007; 151: 157-165. 
[13] Xiao-Li Huang, Xin-Ya Niu, Xiao-Ming Xiu, Xue-Xi Yi,, Quantum Stirling heat engine and refrigerator with single and coupled spin systems, Eur. Phys. J. D (2014) 68: 32. doi:10.1209/0295-5075/99/20005.

[14] Azimi M, Chotorlishvili L, Mishra S K, Vekua T, Hübner W, Berakdar J. Quantum Otto heat engine based on a multiferroic chain working substance. New Journal of Physics 2014; 16: 063018. doi: 10.1088/13672630/16/6/063018.

[15] Dalkıran A., Açıkkalp E., Caner N. Analysis of a quantum irreversible Otto cycle with exergetic sustainable index. Physica A 2016; 453: 316-326. doi: 10.1016/j.physa.2016.02.051.

[16] Wang $\mathrm{H}, \mathrm{Wu}$ G, Chen D. Thermal entangled quantum Otto engine based on the two qubits Heisenberg model with Dzyaloshinskii-Moriya interaction in an external magnetic field. Phys. Scr. 2012; 86: 015001. doi:10.1088/0031-8949/86/01/015001.

[17] Hübner W, Lefkidis G, Dong C D, Chaudhuri D. Spin-dependent Otto quantum heat-engine based on molecular substance. Phys. Rev. B 2014; 90: 024401. doi:10.1103/PhysRevB.90.024401.

[18] Lucia U., Açıkkalp E. Irreversible thermodynamic analysis and application for molecular heat engines. Journal Chemical Physics 2017; 494: 47-55. doi.org/10.1016/j.chemphys.2017.07.009.

[19] Ahmadi M.H., Nabakhteh M.A., Ahmadi M.A., Pourfayaz F., Bidi M. Investigation and optimization of performance of nano-scale Stirling refrigerator using working fluid as Maxwell-Boltzmann gases. Physica A:Stat. Mech. Appl. 2017; 483: 337-350. doi: 10.1016/j.physa.2017.04.079.

[20] Ahmadi M.H., Ahmadi M.A., Maleki A., Pourfayaz F., Bidi M., Açıkkalp E. Exergetic sustainability evaluation and multi-objective optimization of performance of an irreversible nano scale Stirling refrigeration cycle operating with Maxwell-Boltzmann gas. Renew. Sustain. Energy Rev. 2017; 78: 80-92. doi.org/10.1016/j.rser.2017.04.097.

[21] Ahmadi M.H., Ahmadi M.A., Maleki A., Pourfayaz F., Bidi M. Entransy analysis and optimization of performance of nano-scale irreversible Otto cycle operating with Maxwell-Boltzmann ideal gas. Chem. Phys. Lett. 2016; 658: 293-302. doi.org/10.1016/j.cplett.2016.06.058.

[22] Ahmadi M.H., Ahmadi M.A., Maleki A., Pourfayaz F. Performance assessment and optimization of an irreversible nano-scale Stirling engine cycle operating with Maxwell-Boltzmann gas. Eur. Phys. J. Plus 2015; $130: 1-13$. doi:10.1140/epjp/i2015-15190-5.

[23] Sadatsakkak S.A., Ahmadi M.H., Ahmadi M.A.. Optimization performance and thermodynamic analysis of an irreversible nano scale Brayton cycle operating with Maxwell-Boltzmann gas. Energy Convers. Manage. 2015; 101:592-605. doi:10.1016/j.enconman.2015.06.004.

[24] Lucia U., Electron-photon Interaction and Thermal Disequilibrium Irreversibility, International Journal of Quantum Foundations 3 (2017) 24 - 30.

[25] Lucia U. Macroscopic irreversibility and microscopic paradox: A Constructal law analysis of atoms as open systems. Scientific Reports 2016; 6: 35792. doi:10.1038/srep35796.

[26] Lucia U., Some considerations on molecular machines and Loschmidt paradox. Chemical Physics Letters 2015; 623:98-100. doi.org/10.1016/j.cplett.2015.01.055.

[27] Lucia U. Quanta and entropy generation. Physica A 2015; 419: 115-121. doi:10.1016/j.physa.2014.10.040.

[28] Angulo-Brown F., An ecological optimization criterion for finite-time heat engines, Journal of Applied Physic, 69, 7465-7469, 1991.

[29] Yan Z. Comment on Ecological optimization criterion for finite-time heat-engines. Journal of Applied Physic. 1993; 73: 3583. doi:10.1063/1.354041.

[30] Qin X., Chen L., Xia S. Ecological performance of four-temperature-level absorption heat transformer with heat resistance, heat leakage and internal irreversibility. International Journal of Heat and Mass Transfer 2014; 114: 252-257. doi:10.1016/j.ijheatmasstransfer.2017.06.064.

[31] Ge Y., Chen L., Qin X. Effect of specific heat variations on irreversible Otto cycle performance. International Journal of Heat and Mass Transfer 2018; 122: $403-409$. doi:10.1016/j.ijheatmasstransfer.2018.01.132

[32] Zhou J., Chen L. , Ding Z. , Sun F. Analysis and optimization with ecological objective function of irreversible single resonance energy selective electron heat engines. Energy 2016; 111: 306-312. doi: 10.1016/j.energy.2016.05.111. 